\title{
Emergency Visits for Oral Anticoagulant Bleeding
}

Andrew I. Geller, $\mathrm{MD}^{7}$, Nadine Shehab, PharmD, $\mathrm{MPH}^{7}$, Maribeth C. Lovegrove, $\mathrm{MPH}^{7}$, Kathleen O. Rose, RN, BSN ${ }^{7,2}$, Nina J. Weidle, PharmD ${ }^{1,3}$, Sandra K. Goring, RN, BSN ${ }^{7,2}$, and Daniel S. Budnitz, MD, MPH

'Division of Healthcare Quality Promotion, Centers for Disease Control and Prevention (CDC), Atlanta, GA, USA; ${ }^{2}$ Northrup Grumman Corporation, Atlanta, GA, USA; ${ }^{3}$ Eagle Global Scientific LLC, Atlanta, GA, USA.

J Gen Intern Med 35(1):371=3 DOI: $10.1007 / \mathrm{s} 11606-019-05391-y$

(C) Society of General Internal Medicine (This is a U.S. government work and not under copyright protection in the U.S.; foreign copyright protection may apply) 2019

$\mathrm{B}$ leeding Related to Oral Anticoagulants: US Emergency Department Visits, 2017

\section{INTRODUCTION}

Warfarin was the mainstay of outpatient antithrombotic management for decades, but in 2010, direct-acting oral anticoagulants (DOACs) were introduced with fixed dosing and without requirements for laboratory monitoring. ${ }^{1}$ Clinical trials suggested lower rates of major bleeding with DOACs than with warfarin. 2,3 The number of patients prescribed DOACs has been increasing, yet the number treated for emergent DOAC-related bleeding in the USA is unknown. ${ }^{4}$

\section{METHODS}

We estimated emergency department (ED) visits for oral anticoagulant-related bleeding using the National Electronic Injury Surveillance System-Cooperative Adverse Drug Event Surveillance (NEISS-CADES) project, an active public health surveillance system based on a nationally representative, sizestratified probability sample of US hospitals. As described previously, trained abstractors record clinician-diagnosed adverse drug events (ADEs) directly from the medical record. ${ }^{5}$ Each NEISS-CADES case is assigned a sample weight based on hospital sampling design and inverse probability of selection. We used the SAS (v9.4) SURVEYMEANS procedure to calculate national estimates of ED visits, with corresponding 95\% confidence intervals (CIs), accounting for sample weights and complex sample design. NEISS-CADES data collection is considered public health surveillance and exempted from IRB review.

Received March 12, 2019

Revised September 10, 2019

Accepted September 10, 2019

Published online October 29, 2019
National estimates of prescriptions dispensed at outpatient retail and long-term care pharmacies were obtained from the 2017 IQVIA National Prescription Audit (NPA). ${ }^{5}$ Rates of ED visits were calculated by dividing the $\mathrm{ED}$ visit estimate (from NEISS-CADES) by dispensed prescription estimates (from NPA). Because of the large sample size, the variance of NPA estimates was considered negligible.

\section{RESULTS}

Based on 3661 surveillance cases, oral anticoagulant-related bleeding caused an estimated 235,651 (95\% CI, 127,032344,270 ) ED visits in 2017, accounting for $15.5 \%$ (95\% CI, $11.7-19.3 \%)$ of all ED visits for ADEs. The proportion of visits involving DOACs-related bleeding increased from 2.3\% (95\% CI, $0.9-3.7 \%$ ) in 2011 to $37.9 \%$ (95\% CI, 32.7-43.1\%) in 2017 (Fig. 1). In 2017, 49.3\% of DOAC-related bleeding visits involved rivaroxaban, $41.8 \%$ involved apixaban, and $8.9 \%$ involved dabigatran (Table 1 ).

In 2017, approximately one-half $(51.1 \%)$ of oral anticoagulant prescriptions filled in outpatient retail and long-term care pharmacies were for DOACs. Accounting for prescribing frequency, in 2017, the estimated rate for DOAC-related bleeding was 55.3 (95\% CI, 24.9-85.7) and for warfarinrelated bleeding, 94.7 visits per 10,000 dispensed prescriptions (95\% CI, 54.7-134.7).

There were no statistically significant differences in patient sex or age among visits for DOACs compared with warfarin. Adults aged $\geq 65$ years were involved in $80.1 \%$ of ED visits for DOAC-related bleeding and $77.3 \%$ of ED visits for warfarin-related bleeding. Among adults aged $\geq 65$ years, DOAC-related bleeding contributed to $11.8 \%$ (95\% CI, 9.1-14.5\%) of ED visits for ADEs from all drugs, while warfarin-related bleeding contributed to $18.6 \%$ (95\% CI, $15.7-21.5 \%$ ). A nominally higher proportion of DOAC bleeding visits involved gastrointestinal bleeding or epistaxis compared with warfarin, and a nominally lower proportion involved central nervous system bleeding, but differences were not statistically significant (Table 1). A similar proportion of bleeding visits resulted in hospitalization for both DOACs and warfarin $(51.1 \%$ vs. $47.3 \%)$. 


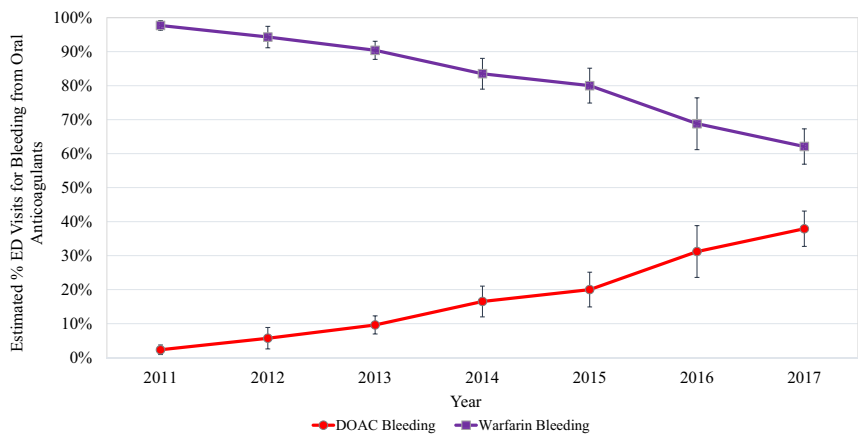

Figure 1 US emergency department visits for oral anticoagulantrelated bleeding, by year, 2011-2017. Estimates from the National Electronic Injury Surveillance System-Cooperative Adverse Drug Event Surveillance project, CDC. Excludes $\boldsymbol{n}=\mathbf{4 0}$ cases implicating both warfarin and a DOAC (2011, $n=2$ cases; 2013, $n=3$ cases; 2014, $n=7$ cases; 2015, $n=8$ cases; 2016, $n=9$ cases; 2017, $n=11$ cases). DOAC, direct-acting oral anticoagulant.

\section{DISCUSSION}

Despite the introduction of a new class of agents with lower rates of major bleeding in clinical trials, anticoagulant-related harm continues to contribute to a large proportion of US ED visits for adverse drug events. Although the rate of DOACrelated bleeding is nominally lower than the rate for warfarin, DOACs are implicated in nearly $40 \%$ of estimated visits for oral anticoagulant-related bleeding, with hospitalization rates comparable with those for warfarin. With further increases in DOAC use, DOAC-related bleeding will likely continue to increase in the future, unless prevention approaches are adopted.

Anticoagulants are essential for the prevention and treatment of thromboembolic disorders and not all anticoagulantrelated bleeding is preventable; however, lessons learned from decades of addressing warfarin-associated bleeding suggest that there may be similar opportunities for DOACs, particularly among older adults who are most vulnerable to anticoagulant ADEs. Future studies could identify a subset of patients who would benefit from specialized management. ${ }^{6}$

These data likely underestimate the number of oral anticoagulant-related bleeds because only events diagnosed and treated in EDs are included. Additionally, events

Table 1 US Emergency Department Visits for Oral Anticoagulant-Related Bleeding, by Case Characteristics, 2017

\begin{tabular}{|c|c|c|c|c|c|c|}
\hline \multirow[t]{4}{*}{ Case characteristic } & \multicolumn{6}{|c|}{ ED visits for bleeding } \\
\hline & \multicolumn{3}{|c|}{ DOAC } & \multicolumn{3}{|c|}{ Warfarin } \\
\hline & \multirow{2}{*}{$\begin{array}{l}\text { Cases } \\
\text { (no.) }\end{array}$} & \multicolumn{2}{|c|}{ National estimate } & \multirow{2}{*}{$\begin{array}{l}\text { Cases } \\
\text { (no.) }\end{array}$} & \multicolumn{2}{|c|}{ National estimate } \\
\hline & & $\%$ & $(95 \% \mathrm{CI})$ & & $\%$ & $(95 \% \mathrm{CI})$ \\
\hline \multicolumn{7}{|l|}{ Patient age group (years) } \\
\hline $0-17$ & 0 & $\sim$ & $\sim$ & 5 & $\sim$ & $\sim$ \\
\hline $18-34$ & 18 & $\sim$ & $\sim$ & 39 & 1.2 & $(0.6-1.8)$ \\
\hline $35-49$ & 66 & 4.5 & $(3.0-5.9)$ & 103 & 4.9 & $(3.9-5.9)$ \\
\hline $50-64$ & 221 & 14.4 & $(12.4-16.5)$ & 367 & 16.4 & $(13.7-19.2)$ \\
\hline $65-79$ & 600 & 44.7 & $(40.9-48.5)$ & 863 & 40.1 & $(37.3-42.9)$ \\
\hline $80+$ & 522 & 35.4 & $(31.0-39.8)$ & 846 & 37.2 & $(33.5-41.0)$ \\
\hline \multicolumn{7}{|l|}{ Patient sex } \\
\hline Female & 712 & 48.8 & $(44.0-53.6)$ & 1019 & 45.9 & $(42.9-48.9)$ \\
\hline Male & 715 & 51.2 & $(46.4-56.0)$ & 1204 & 54.1 & $(51.1-57.1)$ \\
\hline \multicolumn{7}{|l|}{ Drug* } \\
\hline Rivaroxaban & 696 & 49.3 & $(42.3-56.3)$ & & & \\
\hline Apixaban & 617 & 41.8 & $(36.5-47.0)$ & & & \\
\hline \multirow{2}{*}{\multicolumn{7}{|c|}{$\begin{array}{l}\text { Dabigatran } \\
\text { Bleeding manifestation }{ }^{\dagger}\end{array}$}} \\
\hline & & & & & & \\
\hline Central nervous system bleeding & 79 & 4.3 & $(2.3-6.3)$ & 131 & 5.3 & $(4.0-6.7)$ \\
\hline Pulmonary bleeding & 41 & 2.6 & $(1.6-3.7)$ & 65 & 3.1 & $(1.3-4.9)$ \\
\hline Gastrointestinal bleeding & 522 & 40.2 & $(30.5-50.0)$ & 649 & 29.7 & $(23.9-35.6)$ \\
\hline Genitourinary bleeding & 123 & 9.1 & $(5.4-12.8)$ & 189 & 9.0 & $(5.8-12.1)$ \\
\hline Epistaxis & 252 & 21.2 & $(13.1-29.3)$ & 390 & 19.6 & $(14.6-24.6)$ \\
\hline Skin/wound or other minor bleeding & 303 & 17.6 & $(9.6-25.6)$ & 610 & 25.8 & $(18.0-33.5)$ \\
\hline Other types of hemorrhage & 107 & 4.9 & $(2.1-7.7)$ & 189 & 7.5 & $(5.5-9.5)$ \\
\hline \multicolumn{7}{|l|}{ Discharge disposition } \\
\hline Admitted/transferred to another facility & 736 & 48.9 & $(39.6-58.1)$ & 1,063 & 45.5 & $(37.8-53.1)$ \\
\hline Observation admission & 31 & $2.2^{\S}$ & $(0.0-4.5)$ & 47 & $1.9^{\S}$ & $(0.4-3.3)$ \\
\hline Not hospitalized" & 660 & 48.9 & $(40.7-57.0)$ & 1113 & 52.7 & $(45.5-59.9)$ \\
\hline Total & 1427 & & & 2223 & & \\
\hline
\end{tabular}

Case counts and national estimates are from the National Electronic Injury Surveillance System-Cooperative Adverse Drug Event Surveillance project, CDC. Estimates based on $<20$ cases are considered statistically unstable and are not shown $(\sim)$. Excludes $n=11$ cases in which both a DOAC and warfarin were implicated in the ED visit

DOAC direct-acting oral anticoagulant, CI confidence interval

*Not shown: $n=1$ case involving edoxaban-related bleeding

${ }^{\dagger}$ Bleeding manifestations are mutually exclusive and were assigned hierarchically. For example, an ED visit in which a patient experienced both hematemesis and hematuria while on an anticoagulant would be categorized as gastrointestinal bleeding

${ }^{*}$ Includes epidural or subdural hematoma, hemorrhagic stroke, and intracerebral or subarachnoid hemorrhage

${ }^{s}$ Coefficient of variation $>33 \%$

"Defined as treated and released, or left without being seen/left against medical advice 
involving anticoagulants without overt bleeding (e.g., elevated international normalized ratio levels) and fatalities were not included. These data cannot be used for direct comparisons among agents and additional data which may be used for risk adjustment (e.g., current medical comorbidities) are incomplete based on ED documentation.

Acknowledgments: The authors thank Ms. Arati Baral and Mr. Alex Tocitu, from Northrop Grumman (contractor to $\mathrm{CDC}$ ), for assistance with data coding and programming. The authors also thank Mr. Tom Schroeder, Ms. Elenore Sonski, and Mr. Herman Burney, and data abstractors from the US Consumer Product Safety Commission, for their assistance with data acquisition; as well as Dr. Ruth Moro, MD, MPH, for editing assistance. No individuals named herein received compensation for their contributions.

Corresponding Author: Andrew I. Geller, MD; Division of Healthcare Quality Promotion Centers for Disease Control and Prevention (CDC), Atlanta, GA, USA (e-mail: ageller@cdc.gov).

Author Contributions Dr. Geller had full access to all the data in the study and takes responsibility for the integrity of the data and the accuracy of the data analysis.

Study concept and design: Geller, Shehab, Budnitz

Acquisition of data: all authors

Analysis and interpretation of data: all authors

Drafting of the manuscript: Geller, Shehab, Budnitz

Critical revision of the manuscript for important intellectual content: all authors

Statistical analysis: Geller

Administrative, technical, and material support: all authors

Study supervision: Geller, Shehab, Budnitz

Funding Information The funding source was the US federal government.

\section{Compliance with Ethical Standards:}

Federal government employees led or participated in all aspects of the project, including design and conduct of the project; collection, management, analysis, and interpretation of the data; preparation, review, or approval of the manuscript; and decision to submit the manuscript for publication. The findings and conclusions in this study are those of the authors and do not necessarily represent the official position of the Centers for Disease Control and Prevention.

Conflict of Interest: The authors declare that they do not have a conflict of interest.

\section{REFERENCES}

1. Joppa SA, Salciccioli J, Adamski J, et al. A Practical Review of the Emerging Direct Anticoagulants, Laboratory Monitoring, and Reversal Agents. J Clin Med 2018;7(2) https://doi.org/10.3390/jcm7020029.

2. Lip GYH, Banerjee A, Boriani G, et al. Antithrombotic Therapy for Atrial Fibrillation: CHEST Guideline and Expert Panel Report. Chest 2018;154(5):1121-1201. https://doi.org/10.1016/j.chest.2018.07.040.

3. Tritschler T, Kraaijpoel N, Le Gal G, et al. Venous Thromboembolism: Advances in Diagnosis and Treatment. JAMA 2018;320(15):1583-1594. https://doi.org/10.1001/jama.2018.14346.

4. Alalwan AA, Voils SA, Hartzema AG. Trends in utilization of warfarin and direct oral anticoagulants in older adult patients with atrial fibrillation. Am $J$ Health Syst Pharm 2017;74(16):1237-1244. https://doi.org/10.2146/ ajhp160756.

5. Geller AI, Lovegrove MC, Shehab N, et al. National Estimates of Emergency Department Visits for Antibiotic Adverse Events Among Adults-United States, 2011-2015. J Gen Intern Med 2018;33(7):10601068. https://doi.org/10.1007/s11606-018-4430-x.

6. Barnes GD, Nallamothu BK, Sales AE, et al. Reimagining Anticoagulation Clinics in the Era of Direct Oral Anticoagulants. Circ Cardiovasc Bual Outcomes 2016;9(2):182-5. https://doi.org/10.1161/CIRCOUTCOMES. 115.002366 .

Publisher's Note: Springer Nature remains neutral with regard to jurisdictional claims in published maps and institutional affiliations. 